\section{The Evolution of Cognitive Search}

\author{
Thomas T. Hills ${ }^{1}$ and Reuven Dukas ${ }^{2}$ \\ ${ }^{1}$ Department of Psychology, University of Basel, Basel 4055, Switzerland \\ Email: thomas.hills@unibas.ch \\ ${ }^{2}$ Department of Psychology, Neuroscience and Behavior, McMaster University, 1280 \\ Main Street West, Hamilton, Ontario, L8S 4K1, Canada \\ Email:dukas@mcmaster.ca
}

\begin{abstract}
Search can be defined as an attempt to arrive at a goal at an unknown location in the physical environment, time, memory or any other space. Search is necessary because the quantity and quality of resources essential to survival and reproduction vary in space and time. In addition to exploration through actual body movement in their environment, animals also search their external information space through selective allocation of attention and their internal information space in order to retrieve relevant items from memory. Here we integrate data on search in three distinct domains, physical movement, attention to external information, and locating items in memory, in order to highlight the remarkable similarities among these three domains. First, resources in all three domains are typically distributed in patches. Second, in each of the three domains, animals typically keep searching in patches where they have recently found resources and leave areas where they have either not found or depleted resources. Third, the neurobiological mechanisms modulating the exploration for and exploitation of resources in all three domains involve dopamine and, in many vertebrates, regions of the prefrontal cortex and basal ganglia. We suggest that, throughout evolution, animals co-opted existing strategies and mechanisms used to search their physical space for exploring and exploiting internal and external information spaces. The cross disciplinary integration of theory and data about search can help us guide future research on the mechanisms underlying cognitive search.
\end{abstract}


40

41

42

43

44

45

46

47

48

49

50

51

52

53

54

55

56

57

58

59

60

61

62

63

64

65

66

67

68

69

70

71

72

73

74

75

76

77

78

79

80

81

82

83

84

85

\section{INTRODUCTION}

Search is one of the most fundamental of all organismal behaviors. Bacteria seek out essential nutrients and steer clear of noxious compounds (Koshland 1980; Eisenbach \& Lengeler 2004), plant roots search for water and essential nutrients (Hutchings \& de Kroon 1994; McNickle et al. 2009), and the protozoan Paramecium shows in addition to chemotaxis also thermotaxis, geotaxis and thigmotaxis (movement in response to touch) (Jennings 1906; Saimi \&Kung 1987). In general, organisms that move can search for optimal abiotic settings such as temperature, humidity and sunlight, and the best places for finding essential nutrients, avoiding danger and securing sexual partners.

In addition to physically moving through the environment, animals may search within the information space for cues indicating relevant resources. The information space may be external, for example, requiring the direction of attention in pursuit of cues that signal prey, or the information space may be internal, for instance, requiring the directed activation of memory. Whether involving physical movement or not, search involves navigating some space in pursuit of resources. That is, an individual has to decide whether to move (its body or its attention) or stay where it is, and, if it moves, where it should move to. In the domain of physical space, such search problems have been studied extensively in behavioral ecology (Stephens et al., 2007; Stephens \& Krebs, 1986). Research on information search, in both external and internal environments, is developing rapidly (Fu \& Gray, 2006; Hills \& Hertwig, 2010; Pirolli, 2007; Wilke et al., 2009).

In this chapter, we are interested in drawing attention to the potential evolutionary parallels between search across different domains, both external and internal. Why might search in external and internal domains be related in an evolutionary sense? There are three potential forms of evidence. The first is that the neurobiological mechanisms that guide search in different animals may be functionally homologous, deriving from a common ancestral function that was also used to solve search-related problems. The second is that different environments may pose similar kinds of problems for search, generally involving navigating heterogeneous resource distributions to find locations containing resources that maximize fitness. The third form of evidence is that the underlying search strategies share similar characteristics across different environments and domains.

In what follows, we first provide a definition of search, and then we briefly review the three characteristics of environmental structure, search strategies, and neural mechanisms involved in search tasks in external and internal domains. Our review will begin with the physical movement of individuals in space, followed by allocation of attention to external cues, and conclude with search in memory.

\section{What do We Mean by Search?}

Search can be defined as an attempt to arrive at a goal at an unknown location in the physical environment, time, memory or any other space. Finding a resource typically involves at least two components: an exploration phase that investigates possible

All rights reserved by author. No citing, abstracting, or other usage permitted. 
locations where the resource might be located, and an exploitation phase that involves resource acquisition. Often, the exploration and exploitation phases are not mutually exclusive, as animals may sample and exploit during exploration and continue exploring while exploiting.

Because exploration typically takes time away from exploitation, the modulation between the two represents an optimal control problem in which organisms attempt to minimize the time spent exploring for resources but still acquire sufficient information to maximize resource exploitation. When the search task involves a distinct target, an individual only has to choose the optimal movement strategy that would minimize the time needed to find that target. Typically, however, biologically important resources show large variation in quality and they vary over time and space. Thus an adaptive search usually involves a fitness maximizing decision about the optimal balance between exploration and exploitation. More exploration can lead to finding better resources, but less time available for exploiting those resources. This trade-off between exploration and exploitation is common to both external and internal search problems.

\section{EXTERNAL SEARCH: MOVEMENT}

\section{The Structure of the External Resource Environment}

All organisms encounter variation in the quantity and quality of resources. In terrestrial systems, physical factors including the topography, soil types, winds, solar radiation and precipitation shape the spatial structure of temperature and availability of minerals and water. These in turn generate a variable spatial distribution of plant species and of the organisms associated with them. Such distribution may be either continuous or broken, with the latter implying distinct patches varying in the quality and quantity of a given resource each surrounded with regions lacking that resource. Further diurnal and seasonal variation in abiotic factors adds temporal variation in organismal activity and productivity. This combination of spatial and temporal variation in essential abiotic and biotic resources means that an individual's exact location in time and space can dramatically affect its fitness. Hence individuals attempt to optimize their spatial position at any given time.

\section{Search Strategies in External Space}

Confined to the question of physical movement, the central issue concerning search in space is whether or not an organism should stay where it is or move elsewhere. Organisms should make this decision in response to heterogeneity in the density of resources in the surrounding environment. Such problems have been studied extensively (Stephens \& Krebs 1986; Stephens et al., 2007). One approach for examining adaptive 'non-random' foraging behavior involves testing for area-restricted search, which refers to an individual's ability to restrict search to the local area where it has recently found resources before transitioning to more wide-ranging, global exploration (Kareiva \& Odell, 1987). Area-restricted search is related to patch-based models of foraging, like the marginal value theorem (Charnov, 1976), but is often employed when patch boundaries are difficult to detect or otherwise 'fuzzy' (Benhamou, 1992; Adler \& Kotar, 1999). 
132 One of the most primitive forms of search transitions between local and global foraging is

133 the run-and-tumble behavior of bacteria such as Escheria coli. E. coli show a change in

134 behavior upon detecting increasing or decreasing food-concentration gradients 135 (Eisenbach \& Lengeler, 2004; Koshland, 1980). When such resources are increasing, E.

136 coli engage in directed 'runs' of swimming behavior using their flaggelar motor. When

137 resources are decreasing, the direction of the flaggelar motor changes, causing the

138 bacteria to tumble randomly, before engaging in another directed swim. This behavior is

139 mediated by phosphorylation cascades within the bacteria, and appears to serve as a

140 method for moving towards high concentration gradients and away from low

141 concentration gradients. Thus, bacteria show evidence of area-restricted search by

142 attempting to stay in areas with higher resource density, but move away from areas with

143 lower resource density.

144

145

146

147

148

149

150

151

152

153

154

155

156

157

158

159

160

161

162

163

164

165

166

167

168

169

170

171

172

173

174

175

176

177

Figure 1 shows patterns of area-restricted search observed for several classes of animal species: nematodes (Caenorhabditis elegans), house flies (Musca domestica), bumble bees (Bombus bimaculatus), and humans (Homo sapiens). In each case, the central result is that the animal responds to low resource densities by traveling away from them and to high resource densities by staying near them. In the nematode (C. elegans) the animal engages in high angled turns (or pirouettes) following recent encounters with resources, but reduces its number of pirouettes as the time since the last encounter increases (Hills et al., 2004). Similar patterns of increased turning in response to resource encounters are observed in flies (White et al., 1984), bumblebees (Dukas \& Real, 1993), and humans (Kalff et al., 2010). This pattern of density contingent foraging in space is ubiquitous across metazoans (Bell, 1990; Hills, 2006).

\section{Neural Mechanisms of Search in External Space}

What are the neural modulators of spatial search? Despite the abundance of evidence that animals can respond to changing resource densities in space, the neural mechanisms that control this ability are not well understood. Here we focus primarily on dopamine, because other neuromodulators (e.g., norepinephrine and serotonin) are less well understood from a comparative perspective, though they are potentially critical to search and other reward-seeking behaviors (Barron et al., 2010; Cools, this volume).

In nematodes ( $C$. elegans), the modulation between local perseveration and widerranging exploration is governed, at least in part, by a relationship between pre-synaptic dopaminergic neurons modulating downstream glutamatergic locomotory interneurons. Higher levels of dopamine increase turning angles, while lower levels reduce turning angles. Selectively killing dopaminergic neurons or applying a dopaminergic antagonist (raclopride) removes the capacity for area-restricted search (Hills et al., 2004). Dopaminergic mechanisms also facilitate the increased turning that fruit flies (Drosophila melanogaster) show under the influence of cocaine (Bainton et al., 2000), and conditioned place-preference learning in response to methamphetamine in the flatworm, Dugesia japonica (Kusayama \& Watanabe, 2000). In rats (Rattus norvegicus), turning increases in response to agonists for dopaminergic receptors (Robertson \& Robertson, 1986), and modulation between explorative and exploitative behaviors is mediated by midbrain dopaminergic neurons (Fink \& Smith, 1980). In random foraging experiments,

All rights reserved by author. No citing, abstracting, or other usage permitted. 
178 injection of a specific antagonist for the dopaminergic receptor subtype D1 into the

179 nucleus accumbens of rats significantly impaired performance, measured by an increase

180 in wrong entries into maze arms (Floresco \& Phillips, 1999).

181

182

Perseveration in response to resources is known to involve a significant dopaminergic component across animal phyla (for a recent review see Barron et al., 2010). In part, this may be due to the relationship between dopaminergic processing and reward sensitivity.

186 Numerous observations of dopaminergic activity in response to rewards, novel and aversive stimuli have been made, and have given rise to terms like 'reward detector' and 'novelty detector' (Salamone et al., 1997). Critically, dopaminergic neurons adjust their firing rates in response to unpredicted stimuli that are associated with fitness, such as appetitive and aversive stimuli (Salamone et al., 1997). Dopaminergic neurons are also involved in learning to predict outcomes associated with conditioned stimuli (Ljungberg et al., 1992). In vertebrates, the dopaminergic neurons most often associated with goaldirected behaviors are located in the thalamus, striatum, and frontal cortex. These appear to work together to control goal-directed movement in physical space and the focus of attention, which we discuss next.

\section{ATTENTIONAL SEARCH FOR EXTERNAL INFORMATION}

\section{The Structure of the External Information Space}

As noted above, animals encounter non-random distributions of abiotic and biotic resources as they move through their physical environment. This means that the cues indicating the availability and quality of relevant resources including food, predation, potential mates and competitors also show non-random distribution in time and space. Hence individuals can rely on the spatial and temporal structure of certain information for locating resources.

It is obvious that, in many species, searching involves movement in physical space but the issue of search within the external information space is less apparent. Intuitively, one might argue that individuals should just process all incoming relevant information. It is indeed possible that some organisms with very limited perceptual ability can adopt such an inclusive strategy. In animals with extensive perceptual ability, it is clearly optimal to tune out all irrelevant information. Often however, the flow of relevant information exceeds the information processing rate of both the sensory organs and the brain (Dukas, 2002 ; 2009) For example, in humans, only the fovea, which occupies about $0.01 \%$ of the retina and $1.7^{\circ}$ of the visual field, transmits high quality visual information. In primates in general, the optic nerve transmits only approximately $2 \%$ of the information captured by the retinas and only about $1 \%$ of that information is processed by the visual cortex (Van Essen \& Anderson, 1995). In short, an individual's sensory organs can capture only a small proportion of the incoming information flow, and the rate of information capture by the sensory organs far exceeds the brain's rate of information processing. This necessitates a strategy for allocating attention to the most relevant cues in the information space at any given time.

\section{Search Strategies for External Information}


External information can be envisioned as a multidimensional space generated by the information flow from all sense organs. At any given time, animals must choose what information to attend to. This is analogous to the location choices animals make in their physical space (see above). In the information space, animals should attend to the portion of information flow that would have the greatest effect on fitness (Dukas \& Ellner, 1993). For example, when human subjects were more likely to find targets at certain angles of the visual field, they devoted more attention to and had higher detection rates at these angles than subjects searching for randomly distributed targets (Shaw \& Shaw, 1977). Similarly, human subjects tend to focus their visual attention in the vicinity of a recently detected target but switch their attention to other spatial locations if no target is found at this area within a short giving-up time. This behavior, which is reminiscent of arearestricted search, is called inhibition of return (Klein, 2000; Posner \& Cohen, 1984). In general, animals foraging in natural settings should focus their attention on the sensory cues associated with the most profitable food and most likely danger (Dukas, 2002). Whereas much of the research on attention has been done in the visual domain, auditory and olfactory studies have revealed similar patterns of animals focussing on the most relevant cues at any given time (e.g. Skals et al., 2005, Frits and Elhilali 2007, Cross \& Jackson 2010).

Animals searching for resources in the physical environment often have to choose the search rate (distance moved per unit time) that would maximize their rate of finding resources (Dukas, 2002; Gendron \& Staddon, 1983). Similarly, animals have to choose their range of information processing, which should be negatively related to the difficulty of processing certain information (Dukas \& Ellner, 1993). That is, animals can distribute attention broadly (e.g., devote little attention per unit area) when handling easy information but must adopt a narrow focus of attention when handling difficult information. For example, blue jays (Cyanocitta cristata) were trained to search for two prey types, a caterpillar, which could appear in the center of the visual field at a probability of 0.5 , and a moth, which could appear in either right or left peripheries of the visual field at a probability of 0.25 per side. The jays were three times more likely to detect the peripheral moth targets when the central caterpillar was conspicuous, i.e., easy to detect, than when it was cryptic and hence difficult to detect. This result is consistent with the prediction that the jays would process information from the whole visual field when the primary task is easy, but would narrow down their focus of attention to the center field when the primary task is difficult (Dukas \& Kamil, 2000). That is, the jays modulated their focus of attention, reducing the area from which they processed information when the task became more difficult.

\section{Neural Mechanisms Controlling Attention to External Information}

Exactly as dopamine is a key neuromodulator of search in physical space, it plays an important role in search within the external information space. In general, dopamine is involved in subjects' ability to focus and sustain attention on relevant cues. For example, mice (Mus musculus) that were genetically manipulated to selectively eliminate phasic firing of dopaminergic neurons showed selective impairment in using relevant cues for learning. This suggests that the phasic firing of dopaminergic neurons modulates selective attention to relevant information (Caron \& Wightman, 2009; Zweifel et al., 
2009). In humans, subjects with a subtype of the dopamine transporter gene associated with higher dopamine levels in the striatum (a region of the brain associated with attention) show a different pattern of inhibition of return than control subjects (Colzato, Pratt, \& Hommel, 2010). This suggests involvement of dopamine in the spatial allocation of attention over time.

Dopamine deficit is currently the leading theory for explaining attention-deficit / hyperactivity disorder (ADHD), a mental disorder characterized by a reduced ability to focus and sustain attention and by an excessive level of activity. First, brain imaging studies indicate smaller sizes and lesser activation of brain regions related to dopamine in subjects with ADHD. Second, allelic variation in two genes, the dopamine receptor D4 and the dopamine transporter, has been linked to ADHD. Finally, the principal drug for treating ADHD, methylphenidate (Ritalin) increases synaptically released dopamine (Iversen \& Iversen, 2007; Swanson et al., 2007). Together, these examples provide strong evidence that dopamine modulates the focus of attention to external information similar to the way it modulates perseverative local foraging in external space.

\section{INTERNAL INFORMATION SEARCH}

The previous two sections have focused on search via physical movement in the environment and through selective tuning to external information. This section concerns search either for information in memory or for solutions to problems that require internal manipulation of information.

\section{The Structure of Internal Information}

As demonstrated above, external stimuli often present themselves in a non-random, spatially auto-correlated fashion - with rewards associated with a specific location likely to signal rewards close to that location in the near future. Does memory and the structure of the relationships between items in memory implicate an auto-correlated structure, and do we see evidence of this structure in recall from memory?

Studies of written language using network analysis find evidence for a strongly clustered environment. With nodes representing words and links representating relations between words, these networks often show small-world structure, indicating that in text, words are much more likely to appear together in small clusters of related items than one would expect by chance (Cancho \& Solé, 2001). Similar small-world structure has also been identified in internal search when people are asked to say the first word that comes to mind after hearing another word (i.e., free association) (Steyvers \& Tenenbaum, 2005). Moreover, this structure of language and free association networks is well correlated with the order in which children learn about language (Hills et al., 2010a). This indicates that the patchy internal structure of memory may be tightly linked with the patchy external structure of information.

\section{Search Strategies for Internal Information}

Research on free recall from natural categories and list learning consistently find that groups of semantically similar words are produced together (Bousfield, 1953; Romney et al., 1993). This clustering in output is often considered to be the result of a dynamic 
search process that modulates between local and global search policies. One of the most prominent and successful memory search models, the search of associative memory model, employs this dynamic local-to-global search policy (Raaijmakers \& Shiffrin, 1981). Local search is assumed to occur via item level similarity, e.g., with recently recalled items in memory activating other related items in memory. Global search activates all items in relation to the overarching category and context (e.g., "animals" in "Say all the animals you can think of"). Transitions from local to global search occur when local resources become depleted, e.g., when the participants recover too many items that they have already recovered. Interestingly, this model of memory search was developed in cognitive psychology independent of models in behavioral ecology, but it shares the signature behavioral pattern associated with area-restricted search in physical space: modulating between exploration and exploitation in response to recent experience with the resource environment.

Similar evidence for local perseveration due to memory activation has been found in experiments based on word priming. In these experiments, a person is first shown a word-prime (e.g., BIRD) and then asked to determine whether or not a second wordtarget was a true word or a nonword (e.g., ROBIN or ROLIN, respectively). Relative to an uninformative word-prime, Neely (1977) demonstrated both facilitation (faster response times) and inhibition (slower response times) in people's ability to determine the identity of the word-target by manipulating whether the word-target was expected or unexpected following the word-prime. This elegantly demonstrates that expectations create local activation in memory following the presentation of a prime, and that these can reduce the time it takes to recognize objects associated with those memories, but also increase the time it takes to recognize objects that are not associated with those memories.

Research on sequential solutions in problem-solving tasks also demonstrates that people show local perseveration in internal search environments. For example, people tend to produce solutions that are more clustered together (i.e., similar) than one would expect by random generation, for example, in math search tasks (Hills, 2010), and anagram search tasks (Hills et al., 2010b). In one case, Hills et al. (2010b) had participants search within scrambled sets of letters for multiple words. Participants would see a letter set like BLNTAO, and they could find "BOAT", "BOLT", etc. An analysis of the string similarity (e.g., bigram similarity comparing the number of shared letter pairs: 'BO', 'OA', etc.) between subsequent solutions determined that participants tended to produce solutions that were most similar to their last solution. This was true even though previous solutions were not visible. The results indicate that participants were searching locally around previous solutions, before transitioning to a global search strategy (Figure 2).

\section{Neural Mechanisms in Internal Information Search}

Several studies have found that the trajectories taken through long-term memory are related to working memory span (Rosen \& Engle, 1997), which is well known to be tightly connected with dopaminergic processing (Cools \& D'Esposito, 2009). Rosen and Engle (1997) found that participants with higher working memory spans tend to produce longer sequences of clustered items in a category fluency task than individuals with lower 
working memory spans. Hills \& Pachur (In prep.) used a social fluency task ("say all the people that you know") and had participants reconstruct the social network over which they were searching. Using semantic memory models, they found that participants with lower working memory spans transitioned more frequently between global and local cues similar to the transition between exploratory and exploitative behavior described above for spatial and attentional foraging.

Cools and D'Esposito (2009) have suggested that a proper balance between prefrontal and striatal dopamine levels is the key modulator of cognitive stability and cognitive flexibility. Kane and Engle (2002) have suggested that it is the cognitive control of attention, i.e., the ability to focus on one subgoal to the exclusion of other, distracting stimuli, that is the underlying factor determining working memory span. They have further suggested that this ability is mediated by prefrontal cortex modulation of activity in other areas of the brain. In other words, individuals with higher working memory spans are better at exploiting local information in internal search, while individuals with lower working memory spans tend to leave patches of local information more readily.

\section{PROSPECTS}

The data we have presented above indicate three central points about external and internal search. First, the environments in which organisms search both externally and internally share similar structural properties - with resources tending to be patchily distibuted. Second, various search strategies often rely on this patchiness to focus search around areas where resources have been found recently, and thus to facilitate resource acquisition based on their non-random distribution. Finally, the neural mechanisms controlling search, especially those involving dopamine, the prefrontal cortex, and the basal ganglia, are often shared across species and search environments.

While the data above help us integrate information about the structure, strategies and mechanisms of search in external and internal environments, we still lack substantial knowledge about the cognitive ecology of search. Below we highlight three key issues requiring further research.

\section{Physical Search as an Evolutionary Precursor of Cognitive Search}

Might the similarity between external physical search and internal information search indicate an origin for goal-directed cognition (i.e., cognitive control) from an evolutionary precursor devoted to spatial foraging and feeding related behaviors? Across metazoans (i.e., vertebrates and invertebrates), we find similar mechanisms modulating physical search for resources (Barron et al., 2010). As outlined above, in vertebrates (especially mammals) we find roughly the same mechanisms modulating search for information. This suggests a potential evolutionary homology between search in physical space and cognitive search, with the derived form broadening the domains of search to information (Hills, 2006). What other evidence would provide support for or against this hypothesis? 
The comparative evolutionary approach to search also raises several other questions. Are different forms of cognitive search domain specific or domain general? Recent research demonstrated priming in humans from external to internal search (Hills et al., 2008), based on empirical data indicating that prior experience in spatial foraging influenced a subsequent search in an 'internal' problem solving task. In this experiment, participants who first searched in a visuospatial task for clustered or diffuse resources, then searched for word solutions in anagrams as if those solutions were more or less clustered, respectively. This may indicate a domain general search process, consistent with our understanding of executive processing in cognition as a method for navigating hierarchical subgoals (Hills et al,. 2010b). Are there other forms of search that might be guided by such a domain general process, or by other domain specific processes (e.g., mate search)?

Do flexible cognitive capacities rely on balancing neuromodulation, similar to the cognitive search trade-off between exploration and exploitation outlined above? Many pathologies of goal-directed behavior (e.g., ADHD, Parkinson's, stereotypes in autism, drug addiction, etc) involve dopamine in a way that would be predicted from the neural control of animal foraging behavior, with more (or less) synaptic dopamine leading to higher (or lower) levels of perseveration and attentional focus (Hills, 2006). Cools and Robbins (2004) have argued that it is a balance between too-high and too-low dopamine levels that generate the "optimal state of mind"-patterns of behavior associated with too much or too little dopamine are consistently inflexible, often being too compulsive or impulsive for the demands of the environment. This flexibility is potentially one of the guiding selective forces in the evolution of the brain, as relatively larger brains appear to confer greater flexibility - an observation called the cognitive-buffer hypothesis (Sol, 2009). Can we better operationalize what flexibility means in terms of searching for information? And what might be the various evolutionary origins of this flexibility?

How do search strategies evolve to conform to different environments, be they differently distributed environments in physical space, or higher dimensional environments like memory or external information? What other selective forces might underlie the evolution of differences across various search strategies? Finally, what forms of evidence would provide more insight into the evolutionary origins of cognitive search, be they spatial or otherwise?

\section{What is the Biological Basis of Cognitive Search?}

In our analysis of the neural mechanisms underlying search, we focused on the common denominator of neuromodulation by dopamine, which, in vertebrates, is localized principally in the prefrontal cortex and basal ganglia. Whereas this shared characteristic of neuromodulation by dopamine is intriguing and deserves further exploration, a fuller examination must also include more specific details about other brain regions, neuromodulators, and patterns of neuronal firings involved in search within each of the distinct spaces discussed here. Do we find further common mechanisms at this deeper level of analysis? Can existing knowledge about biological mechanisms of search within one domain, say selective attention in external space (Knudsen, 2007; Salamone et al., 
451

452

453

454

455

456

457

458

459

460

461

462

463

464

465

466

467

468

469

470

471

472

473

474

475

476

477

478

479

480

481

482

483

484

485

486

487

488

489

490

491

492

493

494

495

496

2007), help us understand mechanisms of search in another area, for example, retrieval from the internal information space?

\section{The Organization of Internal Information}

Whereas we focused here on similarities across search environments and search mechanisms, there are also some important differences. Perhaps the most significant distinction between external and internal search environments is that searchers typically cannot control the distribution of targets in the external environment but may affect the way they store their own information. That is, natural selection may have shaped the architecture of internally stored information in order to maximize some utility such as the speed of recall or the numbers of items recalled. Perhaps existing models and data on search in external space can help us understand the selective pressures and constraints operating on the structure of internal search environments.

\section{How are the Algorithms for Search Shared Across Domains?}

What are the other dimensions along which we can characterize search? Part of the power of search as a paradigm is our ability to use search algorithms in one domain to inform research in other domains. Here we highlighted the trade-off between exploitation and exploration, that is closely aligned with models of patch foraging. Similar search strategies borrowed from behavioral ecology have recently been applied to human information processing, for example, in terms of giving-up rules in problem solving (Wilke et al., 2009) and how the structure of information in the world wide web can facilitate appropriate search policies (Fu \& Pirolli, 2007). But there are potentially other ways to implement search policies and many dimensions along which they may be defined. Given that some characterizations of search, e.g., exploitation versus exploration, better lend themselves to comparative analysis - both across organisms and algorithms - understanding how we define the dimensions of search and characterize different search policies may help us to better integrate our understanding of search and cognitive abilities.

\section{ACKNOWLEDGEMENTS}

We thank Lauren Dukas, Luc-Alain Giraldeau, David Redish, Peter Todd, and an anonymous referee for comments on the ms, and participants of the Ernst Strüngmann Forum for helpful suggestions. Our work has been supported by a grant from the Swiss National Research Foundation (100014 130397/1) (TH) and the Natural Sciences and Engineering Research Council of Canada, Canada Foundation for Innovation and Ontario Innovation Trust (RD).

\section{REFERENCES}

Adler, F.R., \& Kotar, M. 1999. Departure time versus departure rate: How to forage optimally when you are stupid. Evolutionary Ecology Research, 1:411-421.

Bainton, R. J., Tsai, L. T., Singh, C. M., Moore, M. S., Neckameyer, W. S., \& Heberlein, U. 2000. Dopamine modulates acute responses to cocaine, nicotine and ethanol in Drosophila. Current Biology, 10: 187-94. 
497

498

499

500

501

502

503

504

505

506

507

508

509

510

511

512

513

514

515

516

517

518

519

520

521

522

523

524

525

526

527

528

529

530

531

532

533

534

535

536

537

538

539

540

541

542
Barron, A. B., Søvik, E., \& Cornish, J. L. 2010. The roles of dopamine and related compounds in reward-seeking behavior across animal phyla. Frontiers in Behavioral Neuroscience, 4:1-9.

Bell, W. J. 1990. Searching behavior patterns in insects. Annual Review of Entomology, 35: 447-467.

Benhamou, S. 1992. Efficiency of area-concentrated searching behavior in a continuous patchy environment. Journal of Theoretical Biology, 159: 67-81.

Bousfield, W. 1953. The occurrence of clustering in the recall of randomly arranged associates. Journal of General Psychology, 49: 229-240.

Cancho, R. F., \& Solé, R. V. 2001. The small world of human language. Proceedings of the Royal Society of London. Series B: Biological Sciences, 268: 2261-2265.

Caron, M. G., \& Wightman, R. M. 2009. "To learn, you must pay attention." Molecular insights into teachers wisdom, 106: 7267-7268.

Charnov, E. L. 1976. Optimal foraging: The marginal value theorem. Theoretical Population Biology, 9: 129-136.

Colzato, L. S., Pratt, J., \& Hommel, B. 2010. Dopaminergic control of attentional flexibility: Inhibition of return is associated with the dopamine transporter gene (DAT1). Frontiers in Human Neuroscience, 4: 53.

Cools, R., \& D'Esposito, M. 2009. Dopaminergic modulation of flexible cognitive control in humans. Dopamine Handbook, 1: 249-261.

Cools, R., \& Robbins, T. W. 2004. Chemistry of the adaptive mind. Philosophical Transactions. Series A, Mathematical, Physical, and Engineering Sciences, 362: 2871-88.

Cross, F. R. \& Jackson, R. R. 2010. Olfactory search-image use by a mosquito-eating predator. Proceedings of the Royal Society B: Biological Sciences, 277: 3173-3178.

Dukas, R. 2002. Behavioural and ecological consequences of limited attention. Philosophical Transactions of the Royal Society of London. Series B, Biological sciences, 357: 1539-47.

Dukas, R. 2009. Evolutionary biology of limited attention. In Cognitive Biology: Evolutionary and Developmental Perspectives on Mind, Brain, and Behavior, eds, L. Tommasi, L. Nadel, \& M. Peterson, pp. 147-161. Cambridge, MA: MIT Press.

Dukas, R., \& Ellner, S. 1993. Information processing and prey detection. Ecology, 74: 1337-1346.

Dukas, R., \& Kamil, A. C. 2000. The cost of limited attention in blue jays. Behavioral Ecology, 11: 502-506.

Dukas, R., \& Real, L. A. 1993. Effects of recent experience on foraging decisions by bumble bees. Oecologia, 94: 244-246.

Eisenbach, M., \& Lengeler, J. W. 2004. Chemotaxis. London: Imperial College Press.

Fink, J. S., \& Smith, G. P. 1980. Mesolimbicocortical dopamine terminal fields are necessary for normal locomotor and investigator exploration in rats. Brain Research, 199: 359-384.

Floresco, S. B., \& Phillips, A. G. 1999. Dopamine and hippocampal input to the nucleus accumbens play an essential role in the search for food in an unpredictable environment. Psychobiology, 27: 277-286.

Fritz, J. B., Elhilali, M., David, S. V. \& Shamma, S. A. 2007. Auditory attention -focusing the searchlight on sound. Current Opinion in Neurobiology, 17: 437-455.

All rights reserved by author. No citing, abstracting, or other usage permitted. 
543 Fu, W. T., \& Gray, W. D. 2006. Suboptimal tradeoffs in information seeking. Cognitive 544 Psychology, 52: 195-242.

545 Fu, W. T., \& Pirolli, P. 2007. SNIF-ACT: A cognitive model of user navigation on the World Wide Web. Human-Computer Interaction, 22: 355-412.

Gendron, R. P., \& Staddon, J. E. R. 1983. Searching for cryptic prey: the effects of search rate, The American Naturalist, 121: 172-186.

Hills, T. T. 2006. Animal foraging and the evolution of goal-directed cognition. Cognitive Science, 30: 3-41.

Hills, T. T. 2010. Investigating mathematical search behavior using network analysis. In Modeling Student Mathematical Modeling Competencies, eds. R. Lesh, P. L. Galbraith, C. R. Haines, \& A. Hurford, pp. 571-581, Boston, MA: Springer US.

Hills, T. T., Brockie, P. J., \& Maricq, A. V. 2004. Dopamine and glutamate control arearestricted search behavior in Caenorhabditis elegans. The Journal of Neuroscience, 24: $1217-25$.

Hills, T. T., \& Hertwig, R. 2010. Information search in decisions from experience: Do our patterns of sampling foreshadow our decisions? Psychological Science, 21: 1787-1792.

Hills, T. T., Maouene, J., Riordan, B., \& Smith, L. B. 2010a. The associative structure of language: Contextual diversity in early word learning. Journal of Memory and Language, 63: 259-273.

Hills, T. T., Todd, P. M., \& Goldstone, R. L. 2008. Search in external and internal spaces: evidence for generalized cognitive search processes. Psychological Science 19: 802-8.

Hills, T. T., Todd, P. M., \& Goldstone, R. L. 2010b. The central executive as a search process: priming exploration and exploitation across domains. Journal of Experimental Psychology: General, 139: 590-609.

Hutchings, M. J. \& de Kroon, H. 1994. Foraging in plants: the role of morphological plasticity in resource acquisition. Advances in Ecological Research, 25: 159-238.

Iversen, S. D., \& Iversen, L. L. 2007. Dopamine: 50 years in perspective, Trends in Neurosciences, 30: 188-193.

Jennings, H. S. 1906. Behavior of the Lower Organisms. Bloomington: Indiana University Press.

Kalff, C., Hills, T. T., \& Wiener, J. M. 2010. Human foraging behavior: A virtual reality investigation on area restricted search in humans. In Proceedings of the 32nd Annual Conference of the Cognitive Science Society, eds. R. Catrambone and S. Ohlsson, pp. 1-6. Portland, OR: Cognitive Science Society.

Kane, M. J., \& Engle, R. W. 2002. The role of prefrontal cortex in working-memory capacity, executive attention, and general fluid intelligence: an individualdifferences perspective. Psychonomic Bulletin \& Review, 9: 637-671.

Kareiva, P., \& Odell, G. 1987. Swarms of predators exhibit "preytaxis" if individual predators use area-restricted search. American Naturalist, 130, 233-270.

Klein, R. M. 2000. Inhibition of return. Trends in Cognitive Sciences, 4: 138-147.

Knudsen, E. I. 2007. Fundamental components of attention. Neuroscience, 30: 57-78.

Koshland, D. 1980. Bacterial Chemotaxis as a Model Behavioral System. New York: Raven Press. 
588

589

590

591

592

593

594

595

596

597

598

599

600

601

602

603

604

605

606

607

608

609

610

611

612

613

614

615

616

617

618

619

620

621

622

623

624

625

626

627

628

629

630

631

Kusayama, T., \& Watanabe, S. 2000. Reinforcing effects of methamphetamine in planarians. Neuroreport, 11: 2511-3.

Ljungberg, T., Apicella, P., \& Schultz, W. 1992. Responses of monkey dopamine neurons during learning of behavioral reactions. Journal of Neurophysiology, 67: 145-63.

McNickle, G. G., St. Clair, C. C. \& Cahill Jr, J. F. 2009. Focusing the metaphor: plant root foraging behaviour. Trends in Ecology \& Evolution, 24: 419-426.

Neely, J. 1977. Semantic priming and retrieval from lexical memory: Roles of inhibitionless spreading activation and limited-capacity attention. Journal of Experimental Psychology: General, 106: 226-254.

Pirolli, P. 2007. Information Foraging Theory: Adaptive Interaction with Information. New York, NY: Oxford University Press.

Posner, M. I., \& Cohen, Y. 1984. Components of visual orienting. In Attention and Performance X: Control of Language Processes, eds. H. Bouma \& D. Bouwhuis, pp. 531-556. Hillsdale, NJ: Erlbaum.

Raaijmakers, J. R., \& Shiffrin, R. 1981. Search of associative memory. Psychological Review, 88: 93-134.

Robertson, G. S., \& Robertson, H. A. 1986. Synergistic effects of D1 and D2 dopamine agonists on turning behaviour in rats. Brain Research, 384: 387-90.

Romney, A., Brewer, D., \& Batchelder, W. H. 1993. Predicting clustering from semantic structure. Psychological Science, 4: 28-34.

Rosen, V. M., \& Engle, R. W. 1997. The role of working memory capacity in retrieval. Journal of Experimental Psychology: General, 126: 211-27.

Saalmann, Y. B., Pigarev, I. N., \& Vidyasagar, T. R. 2007. Neural mechanisms of visual attention: How top-down feedback highlights relevant locations. Science, 316: 16121615.

Saimi, Y. \& Kung, C. 1987. Behavioral genetics of paramecium. Annual Review of Genetics, 21: 47-65.

Salamone, J. D., Cousins, M. S., \& Snyder, B. J. 1997. Behavioral functions of nucleus accumbens dopamine: empirical and conceptual problems with the anhedonia hypothesis. Neuroscience and Biobehavioral Reviews, 21: 341-59.

Shaw, M. L., \& Shaw, P. 1977. Optimal allocation of cognitive resources to spatial locations. Journal of Experimental Psychology: Human Perception and Performance, 3: 201-211.

Skals, N., Anderson, P., Kanneworff, M., Lofstedt, C. \& Surlykke, A. 2005. Her odours make him deaf: crossmodal modulation of olfaction and hearing in a male moth. Journal of Experimental Biology, 208: 595-601.

Sol, D. 2009. The cognitive-buffer hypothesis for the evolution of large brains. In Cognitive Ecology II, eds. R. Dukas \& J. M. Ratcliffe, pp. 111-134. Chicago, IL: University Of Chicago Press.

Stephens, D. W., Brown, J. S., \& Ydenberg, R. C. 2007. Foraging: Behavior and Ecology. Chicago, IL: University of Chicago Press.

Stephens, D. W., \& Krebs, J. R. 1986. Foraging Theory. Princeton, NJ: Princeton Univ Press. 
632 Steyvers, M., \& Tenenbaum, J. B. 2005. The large-scale structure of semantic networks:

633 statistical analyses and a model of semantic growth. Cognitive Science, 29: 41-78.

634 Cognitive Science.

635

636

637

638

Swanson, J., Kinsbourne, M., Nigg, J., Lanphear, B., Stefanatos, G., Volkow, N., et al.

639

640

641

642

643

644

645

646

647 2007. Etiologic subtypes of attention-deficit/hyperactivity disorder: brain imaging, molecular genetic and environmental factors and the dopamine hypothesis, 17: 3959.

Van Essen, D. C., \& Anderson, C. H. 1995. Information processing strategies and pathways in the primate visual system. In An Introduction to Neural and Electronic Networks, $2^{\text {nd }}$ ed., eds. S. F. Zornetzer, J. L. Davis, C. Lau, \& T. McKenna, pp. 4576. San Diago: Academic Press.

White, J., Tobin, T., \& Bell, W. J. 1984. Local search in the housefly Musca domestica after feeding on sucrose. Journal of Insect Physiology, 30: 477-487.

Wilke, A., Hutchinson, J., Todd, P. M., \& Czienskowski, U. 2009. Fishing for the right words: Decision rules for human foraging behavior in internal search tasks.

648 Zweifel, L. S., Parker, J. G., Lobb, C. J., Rainwater, A., Wall, V. Z., Fadok, J. P., et al.

649

650

651

652

653

654 2009. Disruption of NMDAR-dependent burst firing by dopamine neurons provides selective assessment of phasic dopamine-dependent behavior, Proceedings of the National Academy of Sciences, 106: 7281-7288. 
A

Nematodes
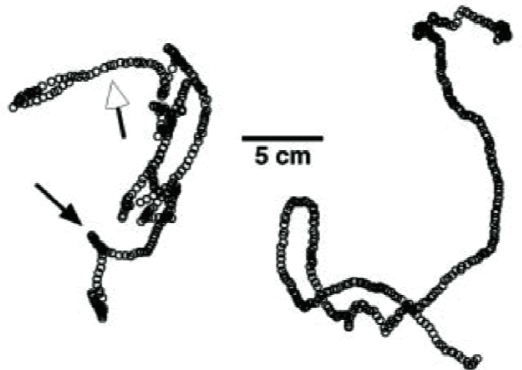

0-5 minutes

30-35 minutes

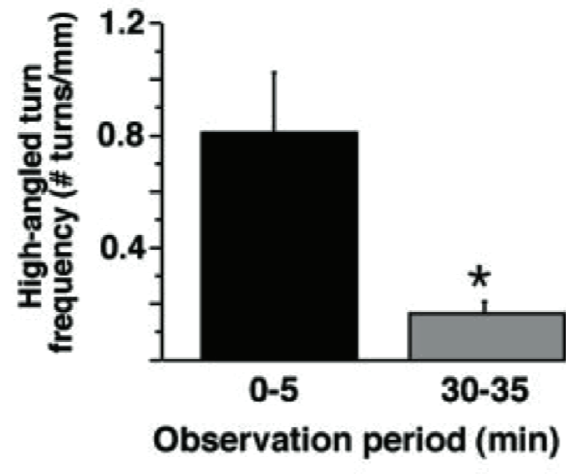

C

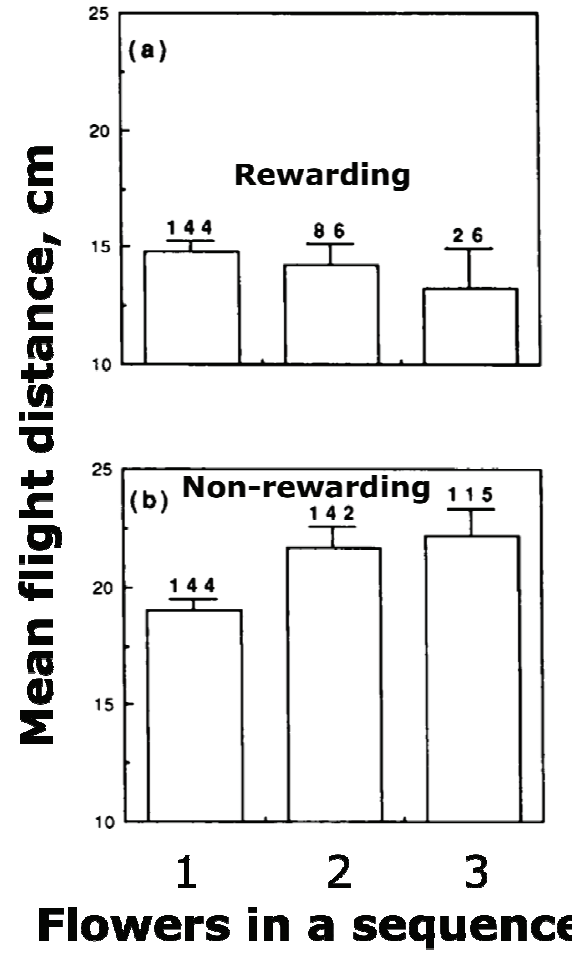

B

House flies
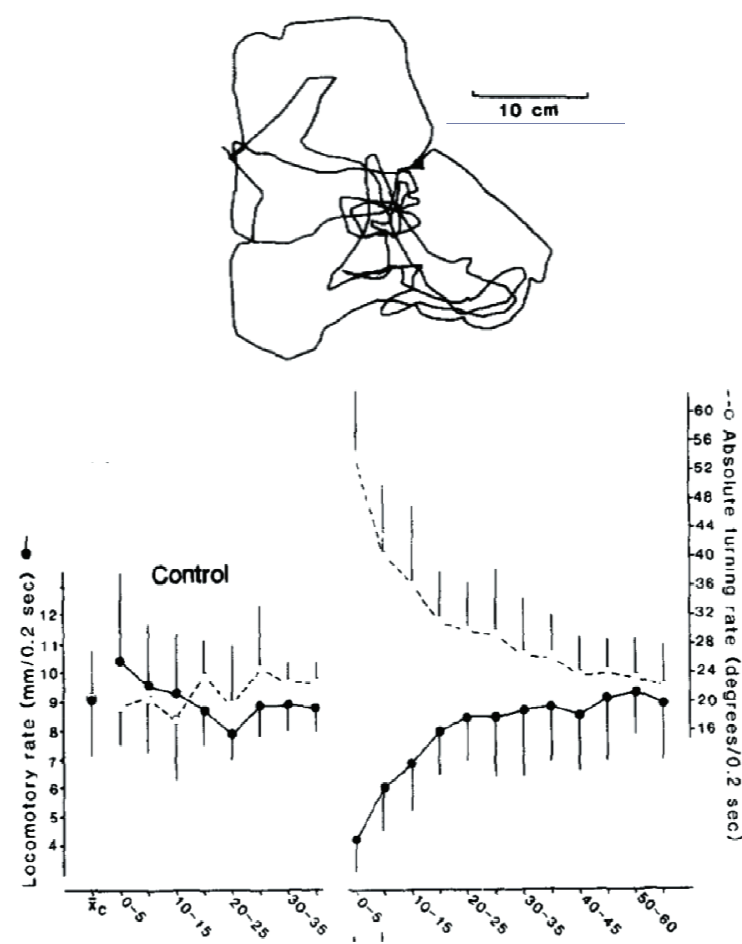

D

Humans
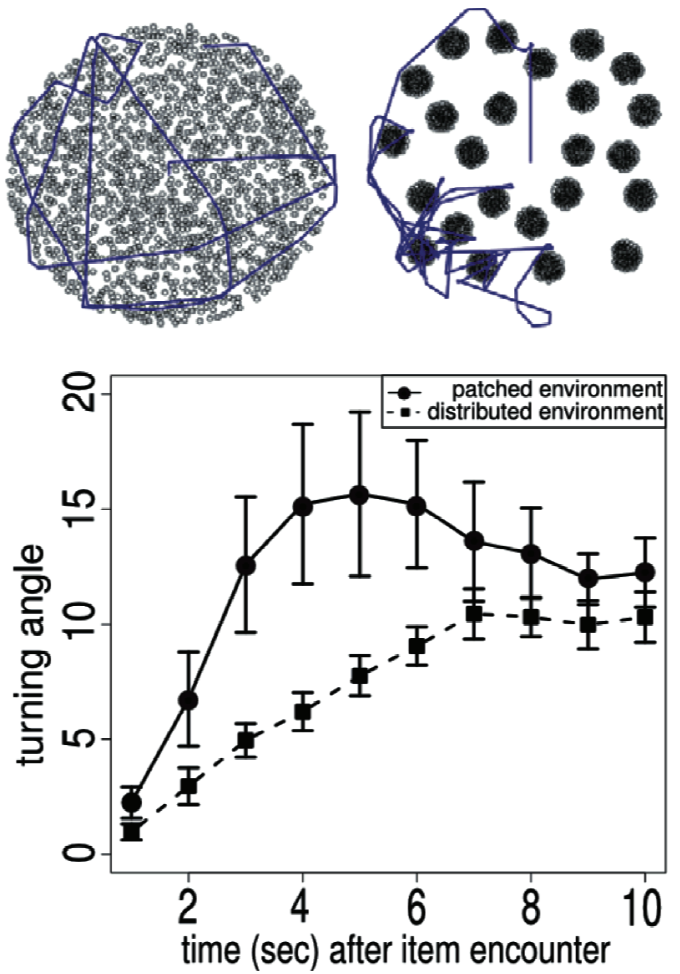
656 Figure 1. Evidence of resource contingent foraging in A) nematodes (Caenorhabditis

657 elegans), B) house flies (Musca domestica), C) Bumble bees (Bombus bimaculatus), and

658 D) humans (Homo sapiens). In A) the top panel shows foraging paths for the nematode

659 C. elegans 0-5 minutes after encountering food and 30-35 minutes later. The filled arrow

660 indicates a high angled turn; the unfilled arrow indicates a region of the path with no

661 turning. The lower panel shows that high angled turns are significantly more likely for

662 the interval more recently associated with food (from Hills et al., 2004). In B) the top

663 panel shows a 69 second path for the house fly M. domestica immediately after

664 encountering food (at the central dot). The lower panel shows the quantitative

665 comparison of turning angle (open circles) and locomotory rate (closed circles) for

666 control flies (on the left) and flies immediately after encountering food (on right) (from

667 White et al., 1984). In C) the top panel shows a significantly decreasing flight distance to

668 the next flower following sequences of one, two, or three rewarding flowers for the

669 bumblebee $B$. bimaculatus. The lower panel shows a significantly increasing flight

670 distance after a series of one, two, or three non-rewarding flowers (data from Dukas \&

671 Real, 1993). In D) the top panel shows typical paths for humans foraging in a 3-

672 dimensional environment with invisible resources arrayed in distributed or clustered

673 arrangements. The lower panel shows that humans show significantly increased turning

674 following encounters with resources in clustered environments than in distributed

675 environments (from Kalff et al., 2010).

676

677 
A

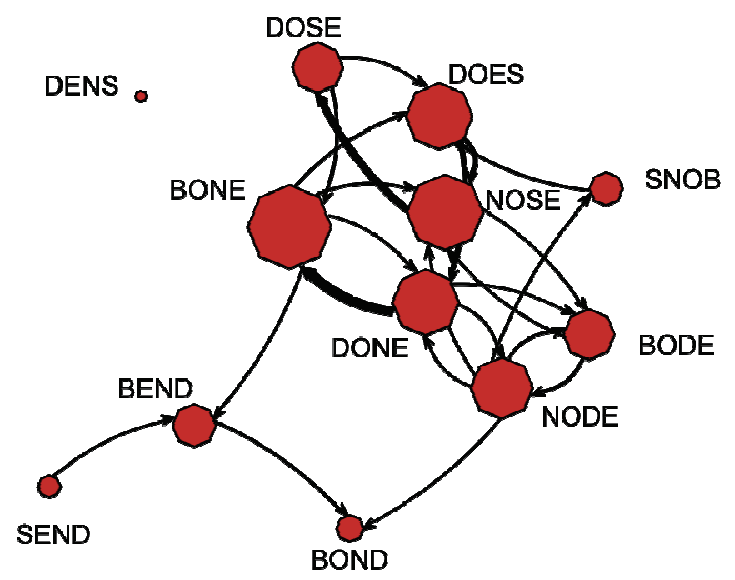

B

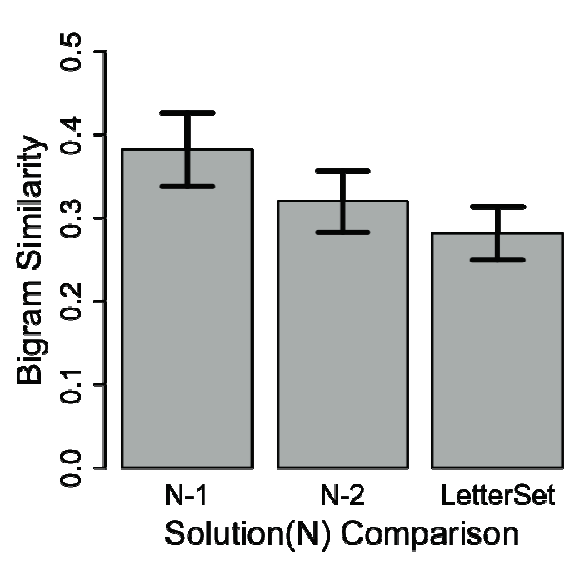

678

679

680

681

682

683

684

685

686

687

688

689

690

691

692

693

694

Figure 2. Behavior in an anagram search task. A. A visual depiction of the between word transitions produced by all participants in the letter set NSBDOE. Participants looked for words they could make from letters in the letter set (using four or more letters). Nodes represent solutions and links between nodes represent transitions between words, with the arrow showing which word came second. Node size is proportional to the number of participants who provided that solution for this letter set. Link thickness is proportional to the number of participants who made that transition. For visual clarity, only transitions that took place more than twice are represented with a link. B. The bigram similarity of the present solution to previous (N-1) and two-back (N-2) solutions and to the original letter set, showing that solutions tended to have the highest string similarity to solutions produced nearby. Error bars are standard error of the mean. Figure borrowed from Hills, Todd, \& Goldstone, 2010. 